\title{
Contextual analysis framework for bursty dynamics
}

\author{
Hang-Hyun Jo, Raj Kumar Pan, Juan I. Perotti, and Kimmo Kaski \\ Department of Biomedical Engineering and Computational Science, Aalto University School of Science, P. O. Box 12200, Espoo, Finland
}

(Received 20 March 2013; published 20 June 2013)

\begin{abstract}
To understand the origin of bursty dynamics in natural and social processes we provide a general analysis framework in which the temporal process is decomposed into subprocesses and then the bursts in subprocesses, called contextual bursts, are combined to collective bursts in the original process. For the combination of subprocesses, it is required to consider the distribution of different contexts over the original process. Based on minimal assumptions for interevent time statistics, we present a theoretical analysis for the relationship between contextual and collective interevent time distributions. Our analysis framework helps to exploit contextual information available in decomposable bursty dynamics.
\end{abstract}

DOI: 10.1103/PhysRevE.87.062131

PACS number(s): 05.40.-a, 89.75.Da, 89.20.-a

\section{INTRODUCTION}

In a wide range of natural and social phenomena, inhomogeneous or non-Poissonian temporal processes have been observed. They are described in terms of $1 / f$ noise [1,2], or in terms of bursts that are rapidly occurring events within short time periods alternating with long periods of low activity [3-5]. In studies of inhomogeneous temporal processes one finds a unified scaling law for the interoccurrence time of earthquakes [6-8], $1 / f$ frequency scaling and power law for interspike interval distributions in neuronal activities $[9,10]$, and heavytailed interevent time distributions in human task execution and communication patterns [3,11-14]. The origin of these temporal inhomogeneities has been extensively investigated in terms of self-organized criticality (SOC) [2,15], where temporal inhomogeneities are a consequence of self-similar structures in temporal patterns. On the other hand, for bursts, other mechanisms have also been suggested, such as memory effects [5] and an inhomogeneous Poisson process with a time-varying event rate [16].

For a more comprehensive understanding of bursty behavior, let us consider a temporal process that can be decomposed into subprocesses. In other words, a set of events with timings comprises events of different contexts, where each context corresponds to each subprocess. For example, communication events of an individual could be classified as being either family related or work related according to the communication partner or content $[17,18]$. We define contextual bursts as bursts of events with the same context, while collective bursts are defined for all kinds of events, i.e., independent of context. Then understanding the contextual bursts can give us more detailed insights into collective bursts. However, the effect of context on bursts has been largely ignored except for a few recent works on human dynamics [19,20]. In order to relate contextual bursts to collective bursts, the distribution of contexts over the original process must be considered in terms of the ordinal time frame, where the real timings of events are replaced by their orders in the original event sequence. The ordinal time frame is useful when the order of events is more

*hang-hyun.jo@aalto.fi crucial for the process than their real timings or when the real timings are not available, such as the sequence of words in the text [21]. In addition, the origin of bursts can be explored more explicitly as the effect of any intrinsic temporal patterns, such as circadian and weekly cycles of humans [22], is excluded. Moreover, the human bursty dynamics has often been modeled in terms of the ordinal time frame by ignoring the real time frame to some extent [3,11,23-25]. Hence, understanding the relation between contextual bursts in real and ordinal time frames is essential for bridging the gap between the models and reality.

In this paper, we provide a general framework for analyzing decomposable bursty dynamics in terms of context and time frame, by studying a minimal model with uncorrelated interevent times. Interestingly, the main part of our model can be translated into the broad class of mass transport models [26,27], although they emerged from totally different backgrounds. We find that the statistical properties of contextual bursts in a real time frame can be dominated by either collective bursts or contextual bursts in an ordinal time frame, or can be characterized by both. We also show that the real and ordinal time frames are related successively by means of deseasoning such that the real time frame is dilated (contracted) for the moment of high (low) activity [22].

The paper is organized as follows. In Sec. II, we devise and analyze the model with uncorrelated interevent times to investigate the relationship between interevent time distributions for collective and contextual bursts in real and ordinal time frames. In Sec. III, we apply the deseasoning method to successively relate the real and ordinal time frames. Finally, we summarize the results in Sec. IV.

\section{MODEL}

Let us now introduce an uncorrelated interevent time model. We denote the collective interevent time by $l$, whereas contextual interevent times in real and ordinal time frames are denoted by $\tau$ and $n$, respectively (see Fig. 1). Their corresponding distributions are written as $P(l), P(\tau)$, and $P(n)$. In general, the contextual real interevent time is obtained by the sum of consecutive collective interevent times, $\tau=$ $\sum_{i=1}^{n} l_{i}$. By means of this relation, the three interevent time 


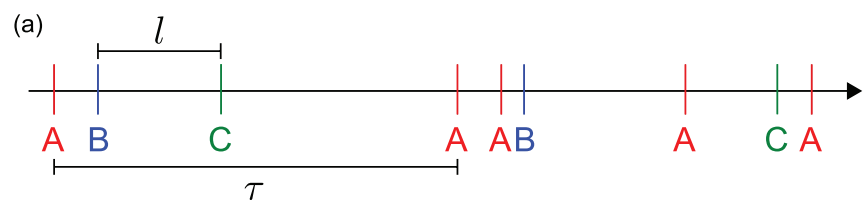

(b)

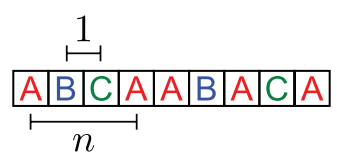

FIG. 1. (Color online) An example of event series with various contexts $\mathrm{A}, \mathrm{B}$, and $\mathrm{C}$, presented in (a) a real time frame and in (b) an ordinal time frame. $l$ and $\tau$ represent the collective and contextual real interevent times, respectively. $n$ represents the contextual ordinal interevent time, while every collective ordinal interevent time is trivially 1 .

distributions are interrelated as follows:

$$
\begin{gathered}
P(\tau)=\sum_{n=1}^{\infty} P(n) F_{n}(\tau), \\
F_{n}(\tau) \equiv \prod_{i=1}^{n} \int_{l_{0}}^{\infty} d l_{i} P\left(l_{i}\right) \delta\left(\tau-\sum_{i=1}^{n} l_{i}\right) .
\end{gathered}
$$

Here $F_{n}(\tau)$ is the probability of obtaining $\tau$ as the sum of $n l \mathrm{~s}$, each of which is independently drawn from the same distribution $P(l)$. Since only one event can occur at a time in our setup, $l$ must have a positive lower bound, $l_{0}>0$. When the variance or tail of $P(l)$ is small, one can approximate $\tau=\sum_{i=1}^{n} l_{i} \approx n\langle l\rangle$ for sufficiently large $n$, where $\langle\cdot\rangle$ denotes an average. This leads to the trivial solution $P(\tau) \approx P(n)$, implying irrelevance of the time frame. As the general case, we consider the heavy-tailed distribution $P(l) \propto l^{-\alpha}$ with $\alpha>$ 1. The distribution of $P(n)$ is closely related to the context distribution over the event sequence. For the case with very few contexts, as $n$ is mostly 1 , i.e., $\tau=l$, we obtain $P(\tau) \approx P(l)$, implying irrelevance of the context. In general we assume that the contexts are unevenly distributed over the event sequence by considering $P(n) \propto n^{-\beta}$ with $\beta>1$. Then we find that $P(\tau)$ shows an asymptotic power-law behavior, $\tau^{-\alpha^{\prime}}$.

\section{A. Main results}

In Fig. 2, we depict the main results. Both collective bursts and contextual bursts in an ordinal time frame are generally expected to affect contextual bursts in a real time frame. This is the case only when both kinds of bursts are sufficiently strong, i.e., $\alpha^{\prime}=(\alpha-1)(\beta-1)+1$ for $\alpha<2$ and $\beta<2$. This scaling relation can be understood by the identity $P(\tau) d \tau=P(n) d n$ with the fact that $\tau=\sum_{i=1}^{n} l_{i}$ is dominated by $\max \left\{l_{i}\right\}$ that is proportional to $n^{1 /(\alpha-1)}$ [28]. On the other hand, when $\alpha>2$ and $\alpha>\beta$, it turns out that the same power-law exponent characterizes contextual bursts in both time frames, i.e., $\alpha^{\prime}=\beta$. This implies that the time frame is not relevant to contextual bursts. Finally, when $\beta>2$ and $\beta>\alpha$, we find $\alpha^{\prime}=\alpha$, implying that the context distribution over the event sequence is not relevant to bursts in a real time frame.

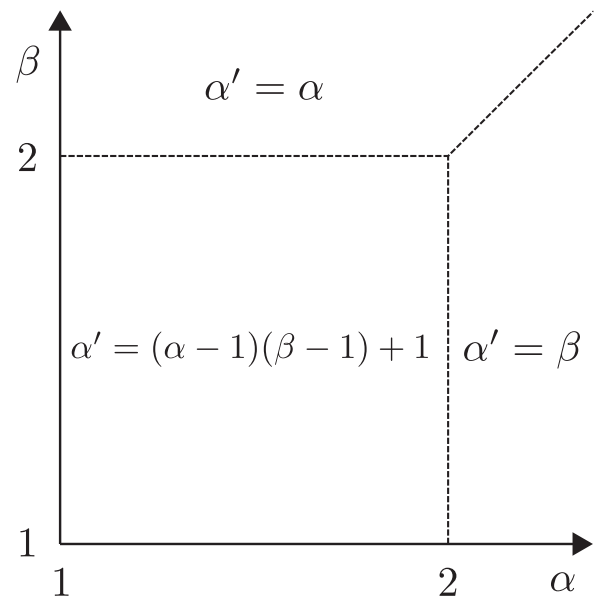

FIG. 2. Phase diagram summarizing the relation between heavytailed distributions of $l, \tau$, and $n$, in terms of their corresponding power-law exponents $\alpha, \alpha^{\prime}$, and $\beta$. Contextual bursts in a real time frame are dominated by contextual bursts in an ordinal time frame if $\alpha>2$ and $\alpha>\beta$, by collective bursts if $\beta>2$ and $\beta>\alpha$, or otherwise characterized by both kinds of bursts.

\section{B. Analysis}

For analysis, we change variables by $m_{i} \equiv l_{i}-l_{0}$ and $M \equiv \tau-n l_{0}$ to rewrite $Z_{n}(M) \equiv F_{n}\left(M+n l_{0}\right)$ and $f\left(m_{i}\right) \equiv$ $P\left(m_{i}+l_{0}\right)$ :

$$
Z_{n}(M)=\prod_{i=1}^{n} \int_{0}^{\infty} d m_{i} f\left(m_{i}\right) \delta\left(M-\sum_{i=1}^{n} m_{i}\right) .
$$

This is exactly the "canonical partition function" for mass transport models and its analytical solution for $f(m) \simeq A m^{-\alpha}$ has been extensively studied [26,27].

For $1<\alpha<2, Z_{n}(M)$ follows a scaling form as [27]

$$
Z_{n}(M) \simeq\left\{\begin{array}{lll}
n^{-v} g_{1}^{-}\left(M n^{-v}\right) & \text { if } \quad M<n, \\
n^{-v} g_{1}^{+}\left(M n^{-v}\right) & \text { if } \quad M>n,
\end{array}\right.
$$

with $v=\frac{1}{\alpha-1}$ and the scaling functions are

$$
\begin{gathered}
g_{1}^{-}(x)=a x^{-\frac{3-\alpha}{2(2-\alpha)}} \exp \left(-b x^{-\frac{\alpha-1}{2-\alpha}}\right), \\
g_{1}^{+}(x)=c x^{-\alpha},
\end{gathered}
$$

where $a, b$, and $c$ are constants depending on $\alpha$ and $A$ [29]. After plugging this scaling form into Eq. (1), we perform the summation over $n$ with the upper bound of $\frac{\tau}{l_{0}}$ due to $M \geqslant 0$. Then, we get

$$
\begin{aligned}
P(\tau)= & \sum_{n=1}^{\tau / l_{0}} P(n) Z_{n}\left(\tau-n l_{0}\right) \\
\propto & \int_{1}^{n_{\times}} n^{-\beta-v} g_{1}^{+}\left[\left(\tau-n l_{0}\right) n^{-v}\right] d n \\
& +\int_{n_{\times}}^{\tau / l_{0}} n^{-\beta-v} g_{1}^{-}\left[\left(\tau-n l_{0}\right) n^{-v}\right] d n \\
\propto & \tau^{-\alpha_{c}}\left[\int_{0}^{x_{\times}} x^{\alpha_{c}-1} g_{1}^{-}(x) d x+\int_{x_{\times}}^{\tau} x^{\alpha_{c}-1} g_{1}^{+}(x) d x\right],
\end{aligned}
$$


with $\alpha_{c} \equiv(\alpha-1)(\beta-1)+1$ and crossovers $n_{\times}$and $x_{\times}=$ $\left(\tau-n_{\times} l_{0}\right) n_{\times}^{-v}$. For derivation, $\left(\tau-n l_{0}\right) n^{-v}$ has been replaced by $x$ and then approximated as $x \approx \tau n^{-v}$. While the first term in the parentheses is independent of $\tau$, the second term is obtained as $\tau^{\alpha_{c}-\alpha}-x_{\times}^{\alpha_{c}-\alpha}$, leading to

$$
P(\tau) \propto c_{1} \tau^{-\alpha_{c}}+c_{2} \tau^{-\alpha},
$$

with coefficients $c_{1}$ and $c_{2}$. Thus, we obtain

$$
\alpha^{\prime}=\min \left\{\alpha_{c}, \alpha\right\} \quad \text { if } \quad 1<\alpha<2 .
$$

The condition for $\alpha_{c}=\alpha$ is $\beta=2$, when the second term in Eq. (6) gives the logarithmic correction as $\ln \tau$. That is, if the
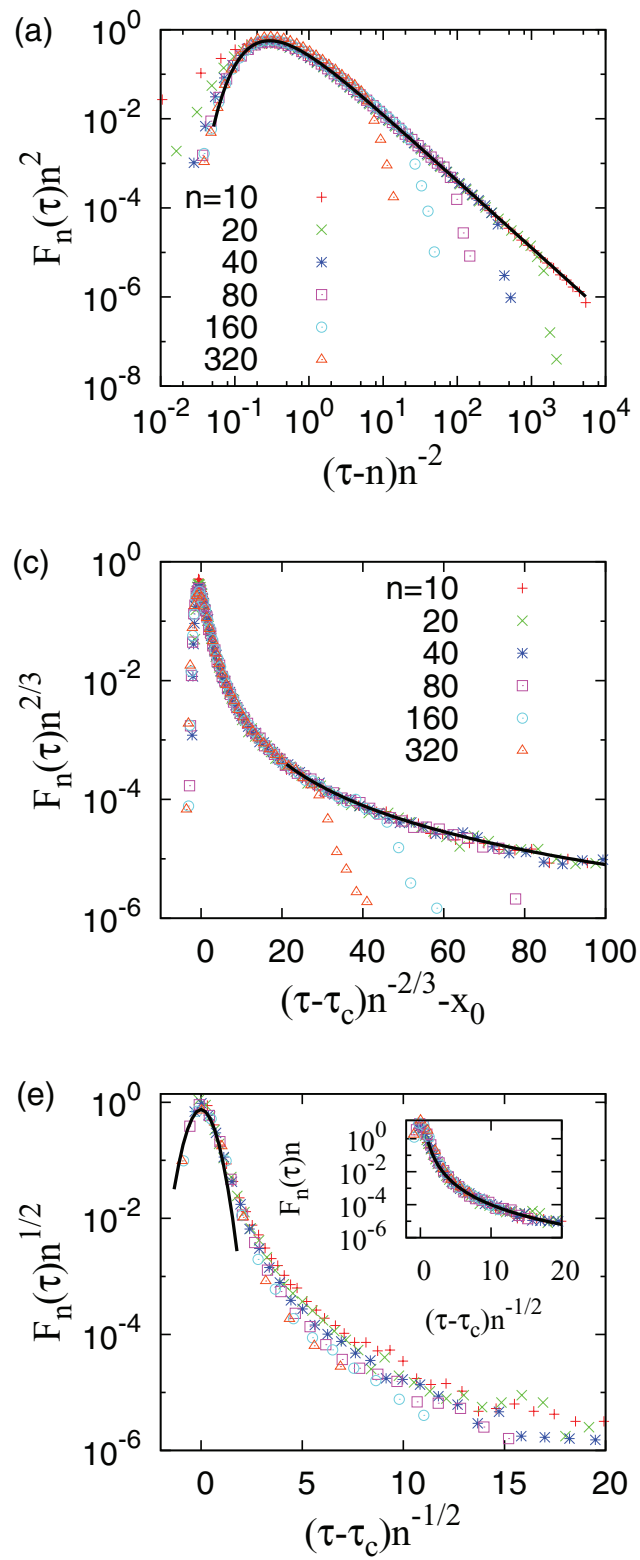

tail of $P(n)$ is sufficiently small, $\alpha^{\prime}=\alpha$ is obtained, implying that contextual bursts in a real time frame are determined only by collective bursts. In any case, we get $\alpha^{\prime}<\beta$, implying that contextual bursts in a real time frame are stronger than those in an ordinal time frame due to large fluctuations of collective interevent times.

Figures 3(a) and 3(b) show that our analysis is confirmed by the numerical simulations (to be described later) for $\alpha=\frac{3}{2}$ and $l_{0}=1$. We find that the numerically obtained $F_{n}(\tau)$ for different $n$ s collapse into one curve corresponding to $g_{1}^{-}(x)$ for $x<x_{\times}$and $g_{1}^{+}(x)$ for $x>x_{\times}$. Then, based on the simple scaling form $P(\tau) \sim \tau^{-\alpha^{\prime}}$, we estimate the value of $\alpha^{\prime}$, which
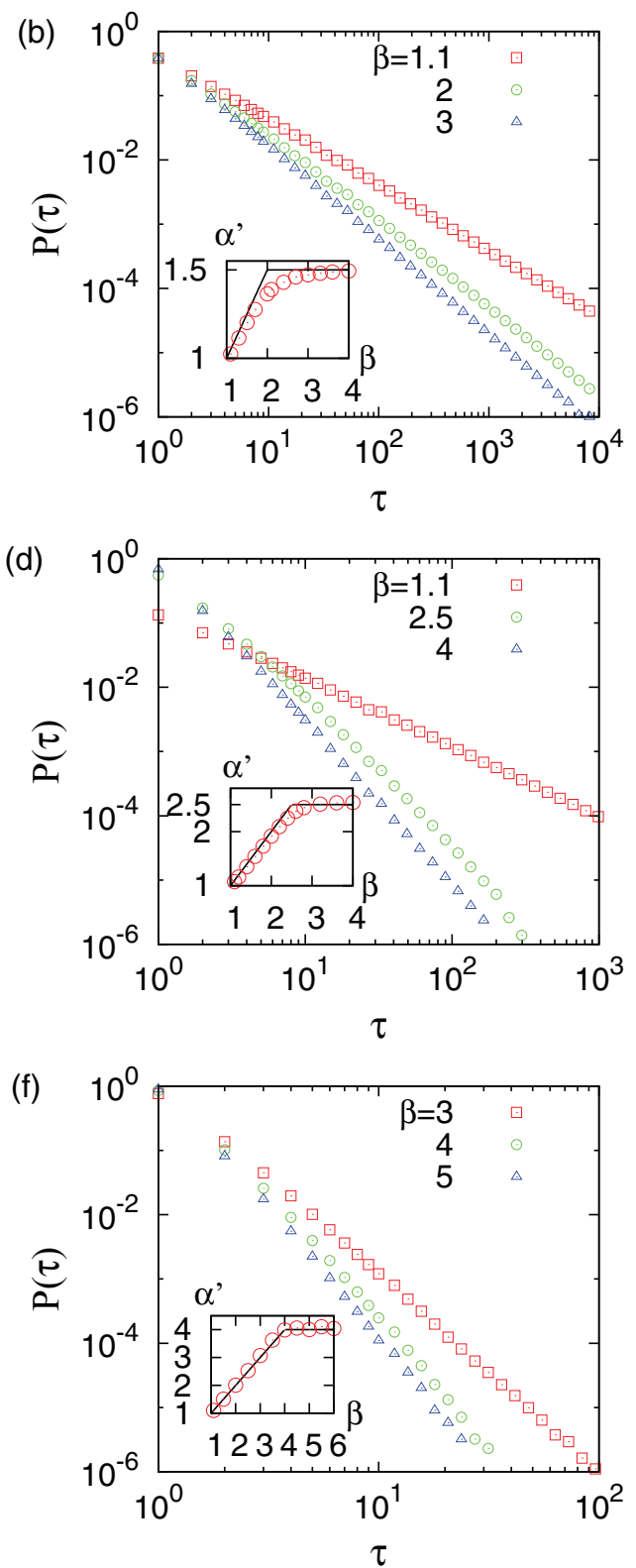

FIG. 3. (Color online) Numerical results of the model for $\alpha=\frac{3}{2}$ (top), $\frac{5}{2}$ (middle), and 4 (bottom), all with $l_{0}=1$. (a) Numerical results of $F_{n}(\tau)$ for different values of $n$ collapsed into one curve, i.e., $g_{1}^{-}(x)$ for $x<x_{\times}$and $g_{1}^{+}(x)$ for $x>x_{\times}$, drawn with a black curve. (b) The power-law exponent $\alpha^{\prime}$ is estimated from $P(\tau) \sim \tau^{-\alpha^{\prime}}$ for each $\beta$ to be compared with the scaling relation $\alpha^{\prime}=\min \left\{\frac{\beta+1}{2}, \frac{3}{2}\right\}$, denoted by a black line in the inset. (c)-(f) Scaling collapse of $F_{n}(\tau)$ and the estimated values of $\alpha^{\prime}$ comparable to $\alpha^{\prime}=\min \{\alpha, \beta\}$ support our analysis for $\alpha>2$. Here $\tau_{c}$ for each $n$ is determined to maximize $F_{n}(\tau)$. In (c) $x_{0} \approx 0.659$ is used. In the inset of (e), tail parts are collapsed by $F_{n}(\tau) n$ vs $\left(\tau-\tau_{c}\right) n^{-1 / 2}$. 
shows a slight discrepancy from the analytic result in Eq. (8), due to the correction term in Eq. (7).

Next, for more realistic considerations such as finite-size data, we discuss the effect of cutoff by assuming that $P(n) \propto$ $n^{-\beta} h\left(\frac{n}{n_{c}}\right)$ with a cutoff function $h(x)$. Let us consider the case of steep cutoff, i.e., $h(x)=1$ for $x \leqslant 1$ and 0 for $x>1$. If $\tau$ is sufficiently large as $\tau>x_{\times} n_{c}^{v}$, we obtain the asymptotic result $\alpha^{\prime}=\alpha$, implying that $\alpha^{\prime}$ is determined only by $\alpha$. In the case of the exponential cutoff $h(x)=e^{-x}$, the same result, $\alpha^{\prime}=\alpha$, is also confirmed by numerical simulations (not shown).

For $2<\alpha<3, Z_{n}(M)$ is a function of $M-M_{c}$ with the "critical point" $M_{c}=n\langle m\rangle$, at which the condensation transition occurs [27]. Thus, we separate the subcritical and supercritical cases as follows:

$$
Z_{n}(M) \simeq\left\{\begin{array}{lll}
n^{-v} g_{2}^{-}\left[\left(M_{c}-M\right) n^{-v}\right] & \text { if } \quad M<M_{c}, \\
n^{-v} g_{2}^{+}\left[\left(M-M_{c}\right) n^{-v}\right] & \text { if } \quad M>M_{c}
\end{array}\right.
$$

where $v=\frac{1}{\alpha-1} \cdot g_{2}^{-}(x)$ has the same form as $g_{1}^{-}(x)$ in Eq. (4), and $g_{2}^{+}(x) \propto x^{-\alpha}$. Since $M-M_{c}=\tau-n\left(l_{0}+\langle m\rangle\right)=\tau-$ $n\langle l\rangle$, we split the summation over $n$ in Eq. (1) at $\frac{\tau}{\langle l\rangle}$ as follows:

$$
\begin{aligned}
P(\tau) \propto & \int_{1}^{\tau /\langle l\rangle} n^{-\beta-v} g_{2}^{+}\left[(\tau-n\langle l\rangle) n^{-\nu}\right] d n \\
& +\int_{\tau /\langle l\rangle}^{\tau / l_{0}} n^{-\beta-v} g_{2}^{-}\left[(n\langle l\rangle-\tau) n^{-v}\right] d n .
\end{aligned}
$$

Similarly to the calculation for $g_{1}^{+}(x)$ in Eq. (6), the first term gives the form of $\tau^{-\alpha_{c}}+\tau^{-\alpha}$. For the second term, we assume that $g_{2}^{-}(x) \approx \delta\left(x-x_{0}\right)$, where the location of peak $x_{0}>0$ is defined by $g_{2}^{-\prime}\left(x_{0}\right)=0$ [30]. Since the root of the equation $\frac{n\langle l\rangle-\tau}{n^{v}}-x_{0}=0$ is in the range of $\left(\frac{\tau}{\langle l\rangle}, \frac{\tau}{l_{0}}\right)$, one finds $\tau^{-\beta}$, leading to $\alpha^{\prime}=\min \left\{\alpha_{c}, \alpha, \beta\right\}$. Knowing that $\beta<\alpha_{c}$ for $\alpha>2$, we find

$$
\alpha^{\prime}=\min \{\alpha, \beta\} \quad \text { if } \quad 2<\alpha<3 .
$$

In other words, collective bursts and contextual bursts in an ordinal time frame compete for contextual bursts in a real time frame. In particular, the result $\alpha^{\prime}=\beta$ for $\beta<\alpha$ implies that the approximation $\tau=\sum_{i=1}^{n} l_{i} \approx n\langle l\rangle$ is still valid even when $\left\langle l^{2}\right\rangle$ diverges. It is because only the subcritical part of $Z_{n}(M)$, where the fluctuation of $l$ is negligible, contributes to $P(\tau)$.

For $\alpha>3, Z_{n}(M)$ can be written by means of central and peripheral scaling functions as [27]

$Z_{n}(M) \simeq\left\{\begin{array}{lll}n^{-v} g_{3}^{<}\left[\left(M-M_{c}\right) n^{-v}\right] & \text { if } & \left|M-M_{c}\right| \lesssim O\left(n^{\frac{2}{3}}\right), \\ n^{-\mu} g_{3}^{>}\left[\left(M-M_{c}\right) n^{-v}\right] & \text { if } \quad M-M_{c} \gtrsim O(n),\end{array}\right.$

where $v=\frac{1}{2}$ and $\mu=\frac{\alpha}{2}-1$. The central scaling function is

$$
g_{3}^{<}(x)=\frac{1}{\sqrt{2 \pi \Delta^{2}}} \exp \left(-\frac{x^{2}}{2 \Delta^{2}}\right),
$$

where $\Delta^{2}=\left\langle m^{2}\right\rangle-\langle m\rangle^{2}$. The peripheral scaling function $g_{3}^{>}(x)$ is the same as $g_{2}^{+}(x)$. By assuming that $g_{3}^{<}(x) \approx \delta(x)$, we obtain $\alpha^{\prime}=\min \{\alpha, \beta\}$. Our analysis is confirmed by the numerical results as shown in Figs. 3(c)-3(f). Finally, all analytical results are summarized as

$$
\alpha^{\prime}=\min \{(\alpha-1)(\beta-1)+1, \alpha, \beta\}
$$

and depicted in Fig. 2.
In our numerical methods, $l$ is considered to be an integer starting from $l_{0}=1$, and so is $\tau$. We prepare a set of collective real interevent times as $L=\left\{1, \ldots, 2, \ldots, l_{\max }\right\}$, where the number of $l$ is proportional to $P(l)=A l^{-\alpha}$ with $A^{-1}=\sum_{l=1}^{l_{\max }} l^{-\alpha}$. Here $l_{\max }$ is determined under the condition $A^{-1}>0.999 \zeta(\alpha)$. When $n$ is given, we randomly select $n$ elements from the set $L$ and get the sum of them as $\tau=\sum_{i=1}^{n} l_{i}$, which is repeated up to $10^{9}$ times to make the distribution $F_{n}(\tau)$. By plugging these $F_{n}(\tau)$ into Eq. (1) together with $P(n)$, we numerically obtain $P(\tau)$.

\section{DESEASONING METHOD}

Although real and ordinal time frames are qualitatively different, we can successively relate them in terms of the deseasoning method [22]. In order to deseason intrinsic cyclic activity, denoted by $\rho(t)$, the real time frame is dilated (contracted) for the moment of high (low) activity. Let us denote the number of events at time $t$ by $a(t)$, being either 0 or 1 in our setup. Given the deseasoning period $T$, the event rate reads

$$
\rho(t)=\frac{T}{s} \sum_{k} a(t+k T)
$$

with the total number of events $s$ and by the normalization $\frac{1}{T} \int_{0}^{T} \rho(t) d t=1$. The deseasoned time $t^{*}$ is defined by means of $\rho^{*}\left(t^{*}\right) d t^{*}=\rho(t) d t$, with $\rho^{*}\left(t^{*}\right)=1$, which implies no cyclic patterns in the deseasoned time frame. Correspondingly, the deseasoned interevent time between event timings $t_{1}$ and $t_{2}$ is defined by $\tau^{*}=\int_{t_{1}}^{t_{2}} \rho\left(t^{\prime}\right) d t^{\prime}$. As the minimum of the deseasoned interevent time is $\frac{T}{s}$, the domain of the deseasoned interevent time distribution becomes smaller for the larger deseasoning period. This means that the deseasoning generically leads to less bursty behavior.

When the time series is fully deseasoned, i.e., $T=T_{f}$ with the entire period $T_{f}$, we get $\rho(t)=\frac{T_{f}}{s} a(t) \equiv l_{0}^{*} a(t)$. Since every collective deseasoned interevent time is $l_{0}^{*}$, the contextual deseasoned interevent time must be a multiple of $l_{0}^{*}$, such that $\tau^{*}=n l_{0}^{*}$. Here $n$ denotes the contextual ordinal interevent time. Conclusively, all temporal properties in the fully deseasoned real time frame should be identical to those in the ordinal time frame. This in turn leads to an interesting question whether contextual bursts in real and ordinal time frames can also be successively related.

\section{SUMMARY}

In summary, we have provided a general framework for analyzing decomposable bursty dynamics in terms of context and time frame, by studying an uncorrelated interevent time model. We derived asymptotic relationships between the collective bursts and contextual bursts in real and ordinal time frames. We found that the contextual bursts in a real time frame can be dominated by either collective bursts or contextual bursts in an ordinal time frame, or be characterized by both kinds of bursts. This implies that collective bursts may have different origins. In particular, the (in)difference 
between the contextual bursts in the real and ordinal time frames is important to relate models in the ordinal time frame with the real systems. Our framework of decomposing a temporal process into subprocesses and combining them after understanding each subprocess helps us to investigate complex systems showing temporal inhomogeneities such as $1 / f$ noise or bursts in more detail. Although temporal inhomogeneities could be understood to some extent only by interevent time distributions, it is important to extend our minimal model to take various correlations and memory effects into account.

\section{ACKNOWLEDGMENT}

Financial support by the Aalto University postdoctoral program (H.J.), and by the Academy of Finland, the Finnish Center of Excellence programme 2006-2011, Project No. 129670 (R.K.P., K.K.), is gratefully acknowledged.
[1] P. Bak, C. Tang, and K. Wiesenfeld, Phys. Rev. Lett. 59, 381 (1987).

[2] P. Bak, How Nature Works: The Science of Self-Organized Criticality (Copernicus, Göttingen, Germany, 1996).

[3] A.-L. Barabási, Nature (London) 435, 207 (2005).

[4] K.-I. Goh and A.-L. Barabási, Europhys. Lett. 81, 48002 (2008).

[5] M. Karsai, K. Kaski, A.-L. Barabási, and J. Kertész, Sci. Rep. 2, 397 (2012).

[6] P. Bak, K. Christensen, L. Danon, and T. Scanlon, Phys. Rev. Lett. 88, 178501 (2002).

[7] A. Corral, Phys. Rev. Lett. 92, 108501 (2004).

[8] J. Davidsen and G. Kwiatek, Phys. Rev. Lett. 110, 068501 (2013).

[9] C. Bédard, H. Kröger, and A. Destexhe, Phys. Rev. Lett. 97, 118102 (2006).

[10] Y. Tsubo, Y. Isomura, and T. Fukai, PLoS Comput. Biol. 8, e1002461 (2012).

[11] A. Vázquez, J. G. Oliveira, Z. Dezsö, K.-I. Goh, I. Kondor, and A.-L. Barabási, Phys. Rev. E 73, 036127 (2006).

[12] U. Harder and M. Paczuski, Physica A 361, 329 (2006).

[13] C. Castellano, S. Fortunato, and V. Loreto, Rev. Mod. Phys. 81, 591 (2009).

[14] Z.-Q. Jiang, W.-J. Xie, M.-X. Li, B. Podobnik, W.-X. Zhou, and H. E. Stanley, Proc. Natl. Acad. Sci. USA 110, 1600 (2013).

[15] M. Paczuski, S. Boettcher, and M. Baiesi, Phys. Rev. Lett. 95, 181102 (2005).

[16] R. D. Malmgren, D. B. Stouffer, A. E. Motter, and L. A. N. Amaral, Proc. Natl. Acad. Sci. USA 105, 18153 (2008).
[17] T. Soikkeli, Master's thesis, Aalto University, 2011.

[18] H.-H. Jo, M. Karsai, J. Karikoski, and K. Kaski, EPJ Data Sci. 1, 10 (2012).

[19] M. Karsai, K. Kaski, and J. Kertész, PLoS One 7, e40612 (2012).

[20] C. Song, D. Wang, and A.-L. Barabási, arXiv:1209.1411.

[21] E. G. Altmann, J. B. Pierrehumbert, and A. E. Motter, PLoS One 4, e7678 (2009).

[22] H.-H. Jo, M. Karsai, J. Kertész, and K. Kaski, New J. Phys. 14, 013055 (2012).

[23] B. Min, K.-I. Goh, and I. M. Kim, Phys. Rev. E 79, 056110 (2009).

[24] H.-H. Jo, R. K. Pan, and K. Kaski, PLoS One 6, e22687 (2011).

[25] H.-H. Jo, R. K. Pan, and K. Kaski, Phys. Rev. E 85, 066101 (2012).

[26] S. N. Majumdar, M. R. Evans, and R. K. P. Zia, Phys. Rev. Lett. 94, 180601 (2005).

[27] M. R. Evans, S. Majumdar, and R. K. P. Zia, J. Stat. Phys. 123, 357 (2006).

[28] L. A. Braunstein, S. V. Buldyrev, R. Cohen, S. Havlin, and H. E. Stanley, Phys. Rev. Lett. 91, 168701 (2003).

[29] Since the saddle point approximation for calculating $g_{1}^{-}(x)$, i.e., Eq. (118) in Ref. [27], is not valid for large $x$, we use the alternative approximation to obtain $g_{1}^{+}(x)$ as described in Appendix A.3.1 in Ref. [27].

[30] Explicitly, $\quad x_{0}=\left[\frac{2(\alpha-2)}{3-\alpha}\right]^{\frac{\alpha-2}{\alpha-1}}[(\alpha-1) A \Gamma(1-\alpha)]^{-\frac{1}{\alpha-1}} \quad$ with $A^{-1}=\int_{0}^{\infty} m^{-\alpha} d m$. Since $A$ is not defined due to the singularity at $m=0$, we use $A \approx \zeta(\alpha)^{-1}$ for the discrete case. For $\alpha=\frac{5}{2}$, $x_{0} \approx 0.659$, which is used in Fig. 3(c). 